PROCEEDINGS OF THE

AMERICAN MATHEMATICAL SOCIETY

Volume 141, Number 1, January 2013, Pages 83-91

S 0002-9939(2012)11300-0

Article electronically published on May 4, 2012

\title{
NEWTON POLYGONS, SUCCESSIVE MINIMA, AND DIFFERENT BOUNDS FOR DRINFELD MODULES OF RANK 2
}

\author{
IMIN CHEN AND YOONJIN LEE
}

(Communicated by Matthew A. Papanikolas)

\begin{abstract}
Let $K=\mathbb{F}_{q}(T)$. For a Drinfeld $A$-module $\phi$ of rank 2 defined over $C_{\infty}$, there are an associated exponential function $e_{\phi}$ and lattice $\Lambda_{\phi}$ in $C_{\infty}$ given by uniformization over $C_{\infty}$. We explicitly determine the Newton polygons of $e_{\phi}$ and the successive minima of $\Lambda_{\phi}$. When $\phi$ is defined over $K_{\infty}$, we give a refinement of Gardeyn's bounds for the action of wild inertia at $\infty$ on the torsion points of $\phi$ and a criterion for the lattice field to be unramified over $K_{\infty}$. If $\phi$ is in addition defined over $K$, we make explicit Gardeyn's bounds for the action of wild inertia at finite primes on the torsion points of $\phi$, using results of Rosen, and this gives an explicit bound on the degree of the different divisor of division fields of $\phi$ over $K$.
\end{abstract}

\section{INTRODUCTION}

Let $K=\mathbb{F}_{q}(T), A=\mathbb{F}_{q}[T]$, where $q$ is a power of a prime $p$, and suppose that $\infty=\left(\frac{1}{T}\right)$ is the place at infinity of $K$ with associated normalized valuation function $v=v_{\infty}: K \rightarrow \mathbb{Z} \cup\{+\infty\}$. Let $K_{\infty}=\mathbb{F}_{q}\left(\left(\frac{1}{T}\right)\right)$ be the completion of $K$ at $\infty$, and $C_{\infty}$ be the completion of an algebraic closure of $K_{\infty}$. Denote also by $v$ the extension of $v$ from $K$ to $C_{\infty}$. Let the absolute value associated to $v$ be given by $|x|=q^{-v(x)}$.

For a specified homomorphism $\iota: A \rightarrow F$, where $F$ is a field, a Drinfeld $A$-module $\phi$ over $F$ is a homomorphism $\phi: A \rightarrow F\langle\tau\rangle$ such that for all $a \in A, \phi_{a}:=\phi(a)$ has constant term $\iota(a)$, where $\tau: z \mapsto z^{q}$ is the $q$-th power Frobenius endomorphism and $F\langle\tau\rangle$ denotes the ring of twisted polynomials over $F$ satisfying $\tau \alpha=\alpha^{q} \tau$ for all $\alpha \in F$. We require that the image of $\phi$ not be contained in $F$. It can be shown that there is an integer $r \geq 1$ called the rank of $\phi$ such that

$$
\phi_{a}=\sum_{i=0}^{r \operatorname{deg} a} a_{i}(\phi, a) \tau^{i}
$$

for all $a \in A$. Note that a Drinfeld $A$-module of rank $r$ is completely determined by its value $\phi_{T}=\iota(T)+\sum_{i=1}^{r} a_{i}(\phi) \tau^{i}$.

Received by the editors April 9, 2011 and, in revised form, June 7, 2011.

2010 Mathematics Subject Classification. Primary 11G09; Secondary 11R58.

Key words and phrases. Drinfeld modules, Newton polygons, exponential functions, minimal bases.

The first-named author was supported by NSERC

The second-named author is the corresponding author and was supported by Priority Research Centers Program through the NRF funded by the Ministry of Education, Science and Technology (2010-0028298) and by the NRF grant funded by the Korea government (MEST) (No. 2011$0015684)$. 
For a Drinfeld $A$-module $\phi$ of rank 2 over $K$, one knows by uniformization (cf. [10]) that there is an $A$-lattice $\Lambda_{\phi}=\Lambda_{\phi, \infty} \subseteq C_{\infty}$ of rank 2 and a surjective analytic function $e_{\phi}=e_{\phi, \infty}: C_{\infty} \rightarrow C_{\infty}$ with zero set equal to $\Lambda_{\phi}$ and such that

$$
e_{\phi}(a z)=\phi_{a} \circ e_{\phi}(z)
$$

for all $a \in A$ and normalized so that the derivative $d e_{\phi}(z) / d z$ of $e_{\phi}(z)$ is equal to 1 . The function $e_{\phi}(z)$ is called the exponential function associated to $\phi$. It is uniquely determined by the above properties and can be written in the form $e_{\phi}(z)=\sum_{i=0}^{\infty} c_{i} \tau^{i}(z)$, where $\tau(z)=z^{q}, c_{i} \in C_{\infty}$, and $c_{0}=1$.

Let $\phi$ be a Drinfeld $A$-module over a field $F$ with respect to a specified homomorphism $\iota: A \rightarrow F$. For any $a \in A, a \neq 0$, we define the $A$-module of $a$-torsion points of $\phi$ as

$$
\phi[a]=\left\{l \in \bar{F} \mid \phi_{a}(l)=0\right\}
$$

and let $F(\phi[a])$ be the field obtained by adjoining the $a$-torsion points of $\phi$ to $F$ (here $\bar{F}$ denotes a fixed algebraic closure of $F$ ).

There has been some interest in studying the field $K(\phi[a])$ generated over $K$ by the $a$-torsion points of $\phi$ [2, 3, 4, 15, 11, 12, 13, 14, 17]. A natural object which arises in bounding the ramification over $\infty$ is the field $K_{\infty}\left(\Lambda_{\phi, \infty}\right)$ which contains the field generated by the $a$-torsion points of $\phi$ over $K_{\infty}$. Since $\Lambda_{\phi, \infty}$ is the zero set of the analytic function $e_{\phi, \infty}(z)$, the different of $K_{\infty}\left(\Lambda_{\phi, \infty}\right) / K_{\infty}$ can be bounded using information from the Newton polygon of $e_{\phi, \infty}(z)$ [4].

In this paper, we explicitly determine the Newton polygon and slopes of $e_{\phi, \infty}(z)$ for a general Drinfeld $A$-module $\phi$ of rank 2 defined over $K$ (in fact, over $C_{\infty}$ ) determined by $\phi_{T}=T+a_{1}(\phi) \tau+a_{2}(\phi) \tau^{2}$. The different cases of Newton polygons which arise depend on $v(j(\phi))$, where $j(\phi)$ is the $j$-invariant of $\phi$, defined by $j(\phi)=$ $a_{1}(\phi)^{q+1} / a_{2}(\phi)$. Some applications of this determination of the Newton polygons are given.

We point out that the essential nature of the Newton polygon of $e_{\phi}$ depends only on the $C_{\infty}$-isomorphism class of $\phi$. Suppose we have an isomorphism $f$ from $\phi$ to $\phi^{\prime}$, where $\phi_{T}=T+a_{1} \tau+a_{2} \tau^{2}$, and $\phi_{T}^{\prime}=T+a_{1}^{\prime} \tau+a_{2}^{\prime} \tau^{2}$. It follows that $f \circ \phi_{a}=\phi_{a}^{\prime} \circ f$ for all $a \in A, f(z)=c z$ for some $c \in C_{\infty}^{*}$, and $a_{i}^{\prime}=a_{i} / c^{q^{i}-1}$. Using Equation (11), we see by induction that $e_{\phi^{\prime}}(z)=\sum_{i=0}^{\infty} c_{i}^{\prime} \tau^{i}(z)=\sum_{i=0}^{\infty} \frac{c_{i}}{c^{q^{i}-1}} \tau^{i}(z)$. It follows that $f \circ e_{\phi}=e_{\phi^{\prime}} \circ f$ and hence $\Lambda_{\phi^{\prime}}=c \Lambda_{\phi}$. Thus, the slopes of the Newton polygon of $e_{\phi^{\prime}}$ are simply the slopes of the Newton polygon of $e_{\phi}$ translated by $-v(c)$, with projected sides having the same lengths and coordinates.

We give explicit bounds on the ramification of $K_{\infty}\left(\Lambda_{\phi, \infty}\right) / K_{\infty}$ at $\infty$ based on a method which slightly refines the one in [4. The ingredient which is needed to make the bound explicit is based on work of Gekeler, which relates $v(j(z))$ and $v(z)$ for $z \in \mathcal{F}$, where $\mathcal{F}=\left\{z \in C_{\infty}:|z|=|z|_{i}=\inf _{x \in K_{\infty}}|z-x| \geq 1\right\}$. In the case of $v(j(\phi)) \geq-q$, the bound does not depend on $\phi$.

Finally, using work of Rosen [15, we make explicit Gardeyn's bounds for the action of wild inertia at finite primes on the torsion points of $\phi$. As a result, we obtain an explicit bound on the degrees of the different divisors of the division fields of $\phi$ over $K$. 


\section{NeWTON POLYGON OF THE EXPONENTIAL FUNCTION ASSOCIATED TO A TWIST OF $\phi$}

We say that $\Lambda \subseteq C_{\infty}$ is an $A$-lattice of rank $r$ if $\Lambda=A \lambda_{1}+\cdots+A \lambda_{r}$ with $\lambda_{i} \in C_{\infty}$ being $K_{\infty}$-linearly independent, and we refer to $\left\{\lambda_{1}, \ldots, \lambda_{r}\right\}$ as an $A$-basis for $\Lambda$.

Let $B_{\kappa}=\{\lambda \in \Lambda:|\lambda| \leq \kappa\}$ for $\kappa \in \mathbb{R}$. We define the $i$ th successive minimum $\nu_{i}$ to be the minimum of the set of $\kappa$ such that $B_{\kappa}$ contains $i$ number of $K$-linearly independent elements for $i=1,2, \ldots, r$, and $\left(\nu_{1}, \ldots, \nu_{r}\right)$ is called the successive minima of $\Lambda$. An ordered $A$-basis $\left(\lambda_{1}, \ldots, \lambda_{r}\right)$ for $\Lambda$ is called a minimal $A$-basis for $\Lambda$ if $\left|\lambda_{i}\right|=\nu_{i}$ for $i=1,2, \ldots, r$ (note: a minimal ordered $A$-basis for $\Lambda$ always exists because of the discreteness of the valuations of elements in $\Lambda$ ).

Lemma 2.1. If $\left\{\omega_{1}, \ldots, \omega_{n}\right\}$ is an A-basis for an A-lattice $\Lambda$ such that $\left|\omega_{1}\right| \leq$ $\left|\omega_{2}\right| \leq \cdots \leq\left|\omega_{n}\right|$, then the following are equivalent:

- $\left(\omega_{1}, \ldots, \omega_{n}\right)$ is a minimal A-basis for $\Lambda$;

- $\left|\sum_{i=1}^{n} a_{i} \omega_{i}\right|=\max \left\{\left|a_{i} \omega_{i}\right|: 1 \leq i \leq n\right\}$ for all $a_{i} \in A$.

Proof. See [18, Lemma 4.2].

By uniformization, we may regard the coefficients $c_{i}=c_{i}(z)$ of $e_{\phi}$ as functions on the upper half-plane $\Omega$, where $\Omega:=C_{\infty}-K_{\infty}$, and $\phi$ is the Drinfeld $A$-module associated to the $A$-lattice $\Lambda=A+A z$. These functions are dubbed the paraEisenstein series by Gekeler [7] and are studied in [6, 8].

Let

$$
\begin{aligned}
\mathcal{F} & =\left\{z \in C_{\infty}:|z|=|z|_{i} \geq 1\right\}, \\
\mathcal{F}_{k} & =\left\{z \in C_{\infty}:|z|=|z|_{i}=q^{k}\right\},
\end{aligned}
$$

where $k \geq 0$ and $|z|_{i}=\inf _{x \in K_{\infty}}|z-x|$.

The subset $\mathcal{F}$ of $\Omega$ is a kind of fundamental domain for $\Omega$ under the action of $\Gamma=\mathrm{GL}_{2}(A)$ in the following sense.

Proposition 2.2. Each element $z \in \Omega$ is $\Gamma$-equivalent to an element of $\mathcal{F}$.

Proof. See [6, Corollary 6.7].

Further properties of $\mathcal{F}$ can be found in [6].

Theorem 2.1. For $z \in \mathcal{F}$ we have the following:

- $\log _{q}|j(z)| \leq q \Longleftrightarrow z \in \mathcal{F}_{0}$.

- Suppose $k \geq 1$. Then $\log _{q}|j(z)|=q^{k+1} \Longleftrightarrow z \in \mathcal{F}_{k}$.

- Suppose $m \geq 1$. Then $m-1<-v(z)<m$ if and only if $q^{m}<-v(j(z))<$ $q^{m+1}$. Furthermore, we have the following linear interpolation property:

$$
-v(z)=\frac{-v(j(z))-q^{m}}{q^{m}(q-1)}+m-1 .
$$

Proof. See [9, Corollary 3.11, Remark 2.4].

Lemma 2.3. If $z \in \mathcal{F}$, then $(1, z)$ is a minimal $A$-basis for the A-lattice $\Lambda=A+A z$.

Proof. Let $z \in \mathcal{F}$. Assume that $(1, z)$ is not a $A$-minimal basis for $A+A z$. Since $|z| \geq 1$, by Lemma 2.1, there exist $a, b \in A$ such that

$$
|a z+b|<\max \{|a z|,|b|\} \text { and }|a z|=|b| \text {. }
$$


It is clear that $a$ must be nonzero. Thus $|z+b / a|<|z|$ and $|z|=|b / a|$. Since $z$ is in $\mathcal{F}$, we have $|z|=|z|_{i}=\inf _{x \in K_{\infty}}|z-x|$. Therefore, $|z| \leq|z+b / a|$. This contradicts the inequality above.

Let $\phi$ be a Drinfeld $A$-module of rank 2 over $C_{\infty}$. Let $\Lambda=\Lambda_{\phi}$ be the $A$-lattice associated to $\phi$ by uniformization, which is the zero set of the exponential function $e_{\phi}$. We know by the fundamental domain property of $\mathcal{F}$ (cf. Proposition 2.2) that $\phi$ is isomorphic over $C_{\infty}$ to another Drinfeld $A$-module $\phi^{\prime}$ such that its exponential function $e_{\phi^{\prime}}$ has associated $A$-lattice $\Lambda^{\prime}=A+A z$, where $z \in \mathcal{F}$.

It suffices to determine the Newton polygon of $e_{\phi^{\prime}}$ as the Newton polygon of $e_{\phi}$ can be deduced from that of $e_{\phi^{\prime}}$ (see Section 3 for further details).

By Lemma 2.3. $(1, z)$ is a minimal $A$-basis for $\Lambda^{\prime}=A+A z$.

2.1. Case 1: $-v(j(\phi)) \leq q$. By Theorem 2.1, this corresponds to the situation $z \in \mathcal{F}_{0}$. The following argument can be found in [6, p. 513]. Since $|z|=1$, we have that $|a z+b|=\max \{|a|,|b|\}$ holds for any $a, b$ in $A$ by Lemma 2.1. This implies that $\Lambda^{\prime}$ has precisely $q^{2(i+1)}$ elements of valuation $\geq-i$, and for each $i \geq 0$, there are $q^{2(i+1)}-q^{2 i}$ elements of valuation $-i$.

Hence, the Newton polygon of $e_{\phi^{\prime}}$ has one segment of length $q^{2 i}\left(q^{2}-1\right)$ of slope $i$ for each $i \geq 0$.

2.2. Case 2 (i): $q<-v(j(\phi)) \neq q^{m+1}$ for any $m \geq 1$. By Theorem 2.1, this corresponds to the situation $q^{m-1}<|z|<q^{m}$ for some $m \geq 1$. Let $|z|=q^{\kappa}$, where $m-1<\kappa<m$. Furthermore, we know that $0<\kappa=-v(z)=\frac{-v(j(\phi))-q^{m}}{q^{m}(q-1)}+m-1$.

Now, by Lemma 2.1 .

$$
|a z+b|=\max \left\{q^{\kappa}|a|,|b|\right\}
$$

holds for any $a, b$ in $A$. In this situation, we see that there are two types of nonzero elements of $\Lambda^{\prime}$ : those with valuation in $-\mathbb{Z}_{\geq 0}$ and those with valuation in $-\left(\kappa+\mathbb{Z}_{\geq 0}\right)$. Thus, the possible slopes of segments of the Newton polygon of $e_{\phi^{\prime}}$, in order, are

$$
0,1, \ldots, m-1, \kappa, m, \kappa+1, m+1, \kappa+2, m+2, \ldots
$$

We now have to count the number of elements of $\Lambda^{\prime}$ of each possible valuation using (2). For $0 \leq i \leq m-1$, there are $q^{i+1}-q^{i}$ elements of valuation $-i$. For $j \geq 0$, there are $q^{m+2 j+1}-q^{m+2 j}$ elements of valuation $-(\kappa+j)$. For $j \geq 0$, there are $q^{m+2 j+2}-q^{m+2 j+1}$ elements of valuation $-(m+j)$.

Hence, the Newton polygon of $e_{\phi^{\prime}}$ has one segment of length $q^{i+1}-q^{i}$ of slope $i$ for each $0 \leq i \leq m-1$ and one segment of length $q^{m+2 j+1}-q^{m+2 j}$ of slope $\kappa+j$, followed by one segment of length $q^{m+2 j+2}-q^{m+2 j+1}$ of slope $m+j$, for each $j \geq 0$.

2.3. Case 2 (ii): $-v(j(\phi))=q^{m+1}$ for some $m \geq 1$. By Theorem 2.1, this corresponds to the situation $|z|=q^{m}$. Thus, as $|a z+b|=\max \left\{q^{m}|a|,|b|\right\}$ holds for any $a, b$ in $A$, there are $q^{i+1}$ elements of $\Lambda^{\prime}$ of valuation $\geq-i$ if $0 \leq i \leq m-1$ and $q^{2 i-m+2}$ elements of $\Lambda^{\prime}$ of valuation $\geq-i$ if $i \geq m$. In particular, for $0 \leq i \leq m-1$, there are $q^{i+1}-q^{i}$ elements of valuation $-i$, and for $i \geq m$, there are $q^{2 i-m+2}-q^{2 i-m}$ elements of valuation $-i$.

Hence, the Newton polygon of $e_{\phi^{\prime}}$ has one segment of length $q^{i}(q-1)$ of slope $i$ for each $0 \leq i<m$ and one segment of length $q^{2 i-m}\left(q^{2}-1\right)$ of slope $i$ for each $i \geq m$. 


\section{NEWTON POLYGON OF $e_{\phi}$ AND VALUATIONS OF SUCCESSIVE MINIMA}

Recall that $\phi$ is a given Drinfeld $A$-module of rank 2 over $C_{\infty}$ with associated exponential function $e_{\phi}$ and $A$-lattice $\Lambda=\Lambda_{\phi}$.

The calculation in Section 2 determined the Newton polygon of $e_{\phi^{\prime}}$, where $\phi^{\prime}$ was a Drinfeld $A$-module isomorphic to $\phi$ over $C_{\infty}$ such that its associated $A$-lattice is of the form $\Lambda^{\prime}=A+A z$ with $z \in \mathcal{F}$.

Since $\Lambda^{\prime}=c \Lambda$ for some $c \in C_{\infty}^{*}$, we know that the slopes of the Newton polygon of $e_{\phi}$ are obtained by translating the slopes of the Newton polygon of $e_{\phi^{\prime}}$ by $v(c)$.

Let $\phi_{T}=T+a_{1} \tau+a_{2} \tau^{2}, \phi_{T}^{\prime}=T+a_{1}^{\prime} \tau+a_{2}^{\prime} \tau^{2}, e_{\phi}=\sum_{i} c_{i} \tau^{i}$, and $e_{\phi^{\prime}}=\sum_{i} c_{i}^{\prime} \tau^{i}$. We have that $a_{i}^{\prime}=a_{i} / c^{q^{i}-1}, c_{i}^{\prime}=c_{i} / c^{q^{i}-1}$, and set $c_{0}=c_{0}^{\prime}=1$ as a normalization.

We know that the first two vertices of the Newton polygon of $e_{\phi}$ are either $(1,0),\left(q, v\left(c_{1}\right)\right)$ in Case 2 or $(1,0),\left(q^{2}, v\left(c_{2}\right)\right)$ in Case 1. In all cases, the slope of the first segment of the Newton polygon of $e_{\phi^{\prime}}$ was 0 .

In Case 2, we have that $v\left(c_{1}\right)=v\left(a_{1}\right)+q$ since $c_{1}=\frac{a_{1}}{T^{q}-T}$, so the slope of the first segment of the Newton polygon of $e_{\phi}$ is $s_{1}=\frac{v\left(a_{1}\right)+q}{q-1}$. The slopes of the remaining segments are those of $e_{\phi^{\prime}}$ translated by $s_{1}$.

On the other hand, in Case 1 , we have that $v\left(c_{2}\right)=v\left(a_{2}\right)+q^{2}$; this is because $c_{2}=\frac{a_{1} c_{1}^{q}+a_{2}}{T^{q^{2}}-T}$ and $v\left(a_{2}\right)<v\left(a_{1} c_{1}^{q}\right)$ in this case. Hence, the slope of the first segment of the Newton polygon of $e_{\phi}$ is $s_{1}=\frac{v\left(a_{2}\right)+q^{2}}{q^{2}-1}$. The slopes of the remaining segments are those of $e_{\phi^{\prime}}$ translated by $s_{1}$.

Putting everything together, we obtain the following theorem.

Theorem 3.1. Let $\phi$ be a Drinfeld A-module of rank 2 over $C_{\infty}$ given by $\phi_{T}=$ $T+a_{1} \tau+a_{2} \tau^{2}$. Let $e_{\phi}$ be its associated exponential function. Let $m$ be the least positive integer such that $-v(j(\phi)) \leq q^{m+1}$. Let $s_{1}=\frac{v\left(a_{2}\right)+q^{2}}{q^{2}-1}$ in Case 1 and $s_{1}=\frac{v\left(a_{1}\right)+q}{q-1}$ in Case 2. Then the Newton polygon of $e_{\phi}$ is determined as follows.

Case 1: $-v(j(\phi)) \leq q$.

The Newton polygon of $e_{\phi}$ has one segment of length $q^{2 i}\left(q^{2}-1\right)$ of slope $i+s_{1}$ for each $i \geq 0$.

Case 2 (i): $q<-v(j(\phi)) \neq q^{m+1}$. Let $\kappa=\frac{-v(j(\phi))-q^{m}}{q^{m}(q-1)}+m-1$. The Newton polygon of $e_{\phi}$ has one segment of length $q^{i+1}-q^{i}$ of slope $i+s_{1}$ for each $0 \leq i \leq m-1$ and one segment of length $q^{m+2 j+1}-$ $q^{m+2 j}$ of slope $\kappa+j+s_{1}$, followed by one segment of length $q^{m+2 j+2}-q^{m+2 j+1}$ of slope $m+j+s_{1}$, for each $j \geq 0$.

Case 2 (ii): $q<-v(j(\phi))=q^{m+1}$.

The Newton polygon of $e_{\phi}$ has one segment of length $q^{i}(q-1)$ of slope $i+s_{1}$ for each $0 \leq i<m$ and one segment of length $q^{2 i-m}\left(q^{2}-1\right)$ of slope $i+s_{1}$ for each $i \geq m$.

Corollary 3.1. Assume the hypotheses and notation of Theorem 3.1. Let $\Lambda$ be the A-lattice associated to $\phi$ by uniformization, and suppose $\left(\lambda_{1}, \lambda_{2}\right)$ is a minimal $A$ basis for $\Lambda$. Then $v\left(\lambda_{1}\right)=-s_{1}=v\left(\lambda_{2}\right)$ if $-v(j(\phi)) \leq q$, and $v\left(\lambda_{1}\right)=-s_{1}, v\left(\lambda_{2}\right)=$ $-s_{1}-\kappa$ if $q<-v(j(\phi)) \leq q^{m+1}$.

Proof. Recall from the proof of Theorem 3.1 that $\Lambda^{\prime}=A+A z=c \Lambda$ for some $z \in \mathcal{F}$, where $c \in C_{\infty}^{*}$, and $(1, z)$ is a minimal $A$-basis for $\Lambda^{\prime}$. Furthermore, it was shown that $v(c)=s_{1}$. 
Now, $\left(\lambda_{1}^{\prime}, \lambda_{2}^{\prime}\right)=\left(\frac{1}{c}, \frac{1}{c} z\right)$ is a choice of minimal $A$-basis for $\Lambda$ since $(1, z)$ is a minimal $A$-basis for $\Lambda^{\prime}=c \Lambda$. Therefore, $v\left(\lambda_{1}^{\prime}\right)=-v(c)=-s_{1}$, and $v\left(\lambda_{2}^{\prime}\right)=$ $-v(c)+v(z)=-s_{1}$ in Case 1 , and $-s_{1}-\kappa$ in Case 2.

Corollary 3.2. Assume the hypotheses and notation of Theorem 3.1 and further that $\phi$ is defined over $K_{\infty}$. Let $\Lambda$ be the A-lattice associated to $\phi$ by uniformization.

If $-v(j(\phi)) \leq q$, then $K_{\infty}(\Lambda) / K_{\infty}$ is unramified if and only if $v\left(a_{2}\right) \equiv-1$ $\left(\bmod q^{2}-1\right)$.

If $q^{m}<-v(j(\phi)) \leq q^{m+1}$ for $m \geq 1$, then $K_{\infty}(\Lambda) / K_{\infty}$ is unramified if and only if $v\left(a_{1}\right) \equiv-1(\bmod q-1)$ and $v(j(\phi)) \equiv-q^{m}\left(\bmod q^{m}(q-1)\right)$.

Remark 3.3. Let $z \in \mathcal{F}_{k}$, where $k \geq 0$, and let $\phi$ be the Drinfeld $A$-module associated to the $A$-lattice $A+A z$, where $\phi_{T}=T+a_{1}(z) \tau+a_{2}(z) \tau^{2}$. The proof of Theorem 3.1 can also be used to give a different proof of the special case $k \leq 0$ of [8, Theorem 2.13], which determines the valuations $v\left(a_{i}(z)\right)$ in terms of $k$ (and $v(j(z))$ if $k=0)$. The idea is to take $\phi=\phi^{\prime}$ in the proof of Theorem 3.1 so that $c=1$, but we also know that $v(c)=s_{1}$, which shows that $v\left(a_{2}(z)\right)=-q^{2}$ in Case 1 and $v\left(a_{1}(z)\right)=-q$ in Case 2. Note, however, that both proofs require Gekeler's theory as a fundamental ingredient.

The case $k=0$ of [8, Theorem 2.13], together with results in [1], can be used to determine the valuations $v\left(c_{i}(z)\right)$ in some new cases not covered in [6].

\section{GARDEYN'S BOUNDS FOR WILD RAMIFICATION AT $\infty$}

Let $\phi$ be a Drinfeld $A$-module of rank 2 defined over $K_{\infty}, e_{\phi}$ its associated exponential function, and $\Lambda_{\phi}=\Lambda_{\phi, \infty}$ its associated $A$-lattice in $C_{\infty}$.

In the following theorem, we give explicit bounds on the ramification of $K_{\infty}\left(\Lambda_{\phi}\right) /$ $K_{\infty}$ based on a slight refinement of the method in [4], which we present in the specific case of rank 2 . We point out that the upper bound of the following theorem is not optimal as Corollary 3.2 shows.

Theorem 4.1. Let $\phi$ be a Drinfeld $A$-module of rank 2 over $K_{\infty}$ and let $\mathcal{D}\left(K_{\infty}\left(\Lambda_{\phi}\right) /\right.$ $\left.K_{\infty}\right)$ be the different of $K_{\infty}\left(\Lambda_{\phi}\right) / K_{\infty}$. Let $m$ be the least positive integer such that $-v(j(\phi)) \leq q^{m+1}$. Then

$$
v\left(\mathcal{D}\left(K_{\infty}\left(\Lambda_{\phi}\right) / K_{\infty}\right)\right) \leq \begin{cases}1 & \text { if }-v(j(\phi)) \leq q, \\ 1+\kappa\left(q^{\kappa+1}-1\right) & \text { if } q<-v(j(\phi)) \leq q^{m+1}\end{cases}
$$

where $\kappa=\frac{-v(j(\phi))-q^{m}}{q^{m}(q-1)}+m-1$.

Proof. Put $\Lambda=\Lambda_{\phi}$, and let $\left(\lambda_{1}, \lambda_{2}\right)$ be a minimal $A$-basis for $\Lambda$ such that $z=$ $\lambda_{2} / \lambda_{1} \in \mathcal{F}$.

For any $s>0$, there exist $d \in C_{\infty}^{*}$ and $\delta$ such that $v(d)=-v\left(\lambda_{2}\right)+\delta$, where $0 \leq \delta<\frac{1}{q^{s}-1}$, and the ramification index of $K_{\infty}^{\prime}=K_{\infty}(d)$ divides $q^{s}-1$.

Let $\Lambda^{0}=A \lambda_{1}^{0}+A \lambda_{2}^{0}$, where $\lambda_{i}^{0}=d \lambda_{i}$. Then $\left(\lambda_{1}^{0}, \lambda_{2}^{0}\right)$ is a minimal $A$-basis for $\Lambda^{0}$ since $\left(\lambda_{1}, \lambda_{2}\right)$ is a minimal $A$-basis for $\Lambda$, and $K_{\infty}^{\prime}\left(\Lambda^{0}\right)=K_{\infty}^{\prime}(\Lambda)$. Let $G_{\Lambda^{0}}$ be the Galois group of $K_{\infty}^{\prime}\left(\Lambda^{0}\right) / K_{\infty}^{\prime}$, and $K_{\infty}^{0}$ be the maximal tamely ramified subextension of $K_{\infty}^{\prime}\left(\Lambda^{0}\right) / K_{\infty}^{\prime}$, corresponding to the Sylow $p$-subgroup $P_{\Lambda^{0}}$ of $G_{\Lambda^{0}}$ (recall that $p=\operatorname{char}(K))$. For $\sigma \in G_{\Lambda^{0}}, \sigma \lambda_{1}^{0}=\alpha \lambda_{1}^{0}$, where $\alpha \in \mathbb{F}_{q}^{*}$, as $\left|\sigma \lambda_{1}^{0}\right|=\left|\lambda_{1}^{0}\right|$. It follows that $\lambda_{1}^{0} \in K_{\infty}^{0}$ and $K_{\infty}^{\prime}\left(\Lambda^{0}\right)=K_{\infty}^{0}\left(\lambda_{2}^{0}\right)$. 
Now, $v\left(\lambda_{2}^{0}\right)=\delta \geq 0$. Using [16, III, Cor. 2, p. 66], we have that

$$
\mathcal{D}\left(K_{\infty}^{0}\left(\lambda_{2}^{0}\right) / K_{\infty}^{0}\right) \mid \prod_{\sigma \in P_{\Lambda^{0}}, \sigma \neq 1}\left(\sigma \lambda_{2}^{0}-\lambda_{2}^{0}\right) .
$$

For $\sigma \in P_{\Lambda^{0}}$ with $\sigma \neq 1$, we have that $\sigma \lambda_{2}^{0}=\beta \lambda_{1}^{0}+\lambda_{2}^{0}$, where $\beta$ is nonzero in $A$ and satisfies $|\beta| \leq\left|\lambda_{2} / \lambda_{1}\right|$ by Lemma 2.1. It follows that $\# P_{\Lambda^{0}} \leq q\left|\lambda_{2}\right| /\left|\lambda_{1}\right|$. Finally, $\left|\sigma \lambda_{2}^{0}-\lambda_{2}^{0}\right|=\left|\beta \lambda_{1}^{0}\right| \geq\left|\lambda_{1}^{0}\right|$, so $v\left(\sigma \lambda_{2}^{0}-\lambda_{2}^{0}\right) \leq v\left(\lambda_{1}^{0}\right)$, and hence

$$
\begin{aligned}
v\left(\mathcal{D}\left(K_{\infty}^{0}\left(\lambda_{2}^{0}\right) / K_{\infty}^{0}\right)\right) & \leq\left(q\left|\lambda_{2} / \lambda_{1}\right|-1\right) v\left(\lambda_{1}^{0}\right) \\
& =\left(q\left|\lambda_{2} / \lambda_{1}\right|-1\right)\left(v\left(\lambda_{1} / \lambda_{2}\right)+\delta\right) .
\end{aligned}
$$

The extension $K_{\infty}^{0} / K_{\infty}$ is tamely ramified, so we obtain that

$$
v\left(\mathcal{D}\left(K_{\infty}(\Lambda) / K_{\infty}\right)\right) \leq 1+v\left(\mathcal{D}\left(K_{\infty}^{0}\left(\lambda_{2}^{0}\right) / K_{\infty}^{0}\right)\right) .
$$

From Corollary [3.1, if $-v(j(\phi)) \leq q$, then $|z|=1$ and $\delta=0$, and if $q<$ $-v(j(\phi)) \leq q^{m+1}$, then $|z|=q^{\kappa}$. Thus, we have

$$
v\left(\mathcal{D}\left(K_{\infty}(\Lambda) / K_{\infty}\right)\right) \leq \begin{cases}1 & \text { if }-v(j(\phi)) \leq q, \\ 1+(\kappa+\delta)\left(q^{\kappa+1}-1\right) & \text { if } q<-v(j(\phi)) \leq q^{m+1},\end{cases}
$$

where $0 \leq \delta<\frac{1}{q^{s}-1}$. Taking $s \rightarrow \infty$ gives the desired bound.

Remark 4.1. For Theorem 4.1, using [4] directly would instead yield a bound of 1 if $-v(j(\phi)) \leq q$, and $1+2 \kappa q^{\kappa+1}$ if $q<-v(j(\phi)) \leq q^{m+1}$.

We notice that in the range of $v(j(\phi)) \geq-q$, the bound on the different of $K_{\infty}\left(\Lambda_{\phi}\right) / K_{\infty}$ does not depend on $\phi$.

\section{GaRdeyn's bounds For Wild RAMification at Finite PRIMES $\mathfrak{p}$}

Let $\mathfrak{p}$ be a finite prime of $K, K_{\mathfrak{p}}$ be the completion at $\mathfrak{p}$, and denote by $v_{\mathfrak{p}}$ its associated valuation. It is well known that $\phi$ has potentially Tate (resp. potentially good) reduction over $K_{\mathfrak{p}}$ if $v_{\mathfrak{p}}(j(\phi))<0\left(\right.$ resp. $\left.v_{\mathfrak{p}}(j(\phi)) \geq 0\right)$ and that the stable reduction occurs over a finite tamely ramified extension of $K_{\mathfrak{p}}$ [15, Lemma 5.2].

By 4, we have that

$v_{\mathfrak{p}}\left(\mathcal{D}\left(K_{\mathfrak{p}}(\phi[a]) / K_{\mathfrak{p}}\right)\right) \leq \begin{cases}2 v_{\mathfrak{p}}(a) & \text { if } \phi \text { has good reduction over } K_{\mathfrak{p}}, \\ 2 v_{\mathfrak{p}}(a)+1 & \text { if } \phi \text { has good reduction over } K_{\mathfrak{p}}^{\prime}, \\ 2 v_{\mathfrak{p}}(a)+1-2 v_{\mathfrak{p}}\left(\lambda_{1}\right) & \text { if } \phi \text { has Tate reduction over } K_{\mathfrak{p}}^{\prime},\end{cases}$

where $K_{\mathfrak{p}}^{\prime}$ is a finite tamely ramified extension of $K_{\mathfrak{p}}$, and $\lambda_{1}$ is defined as follows.

In the case that $\phi$ has Tate reduction over $K_{\mathfrak{p}}^{\prime}$, we obtain from uniformization that there is a Drinfeld $A$-module $\psi$ of rank 1 and a surjective exponential function $e_{\phi, \mathfrak{p}}: C_{\mathfrak{p}} \rightarrow C_{\mathfrak{p}}$ such that $e_{\phi, \mathfrak{p}} \circ \psi_{a}=\phi_{a} \circ e_{\phi, \mathfrak{p}}$ for all $a \in A$, where $C_{\mathfrak{p}}$ is the completion of an algebraic closure of $K_{\mathfrak{p}}$.

The zeroes of $e_{\phi, \mathfrak{p}}$ form an $A$-lattice $\Lambda_{\mathfrak{p}}=\Lambda_{\phi, \mathfrak{p}}$ of rank 1 in $C_{\mathfrak{p}}$, so suppose $\Lambda_{\mathfrak{p}}=A \lambda_{1}$. Note that it is necessarily the case that $v\left(\lambda_{1}\right)<0$ and $\left(\lambda_{1}\right)$ is a minimal $A$-basis for $\Lambda_{\mathfrak{p}}$.

From [15, Lemma 5.3], we have that $v_{\mathfrak{p}}\left(\lambda_{1}\right)=\frac{1}{q-1} v_{\mathfrak{p}}(j(\phi))$.

Combining the above estimations yields the following explicit upper bound for the different $\mathcal{D}\left(K_{\mathfrak{p}}(\phi[a]) / K_{\mathfrak{p}}\right)$. 
Theorem 5.1. Let $\phi$ be a Drinfeld $A$-module of rank 2 over $K_{\mathfrak{p}}$ and let $\mathcal{D}\left(K_{\mathfrak{p}}(\phi[a]) /\right.$ $\left.K_{\mathfrak{p}}\right)$ be the different of $K_{\mathfrak{p}}(\phi[a]) / K_{\mathfrak{p}}$. Then

$$
v_{\mathfrak{p}}\left(\mathcal{D}\left(K_{\mathfrak{p}}(\phi[a]) / K_{\mathfrak{p}}\right)\right) \leq \begin{cases}2 v_{\mathfrak{p}}(a) & \text { if } \phi \text { has good reduction over } \\ & K_{\mathfrak{p}}, \\ 2 v_{\mathfrak{p}}(a)+1 & \text { if } v_{\mathfrak{p}}(j(\phi)) \geq 0 \text { and } \phi \text { has } \\ 2 v_{\mathfrak{p}}(a)+1-\frac{2}{q-1} v_{\mathfrak{p}}(j(\phi)) & \text { bad reduction over } K_{\mathfrak{p}}, \\ \text { if } v_{\mathfrak{p}}(j(\phi))<0 .\end{cases}
$$

For a finite extension $L / K$, let $\mathfrak{D}(L / K)$ be the different divisor of $L / K$ and define the degree with respect to $K$ of $\mathfrak{D}(L / K)$ as

$$
\operatorname{deg}_{K} \mathfrak{D}(L / K)=\sum_{v} \max \left\{v\left(\mathcal{D}\left(L_{w} / K_{v}\right)\right): w \mid v\right\} \operatorname{deg}_{K} v
$$

where $v$ ranges through all normalized places of $K, w$ through all places of $L$ lying over each $v$, and $\mathcal{D}\left(L_{w} / K_{v}\right)$ is the different of $L_{w} / K_{v}$. It can be shown that

$$
\operatorname{deg}_{L} \mathfrak{D}(L / K) \leq n^{\prime} \operatorname{deg}_{K} \mathfrak{D}(L / K),
$$

where $n^{\prime}$ is the geometric extension degree of $L / K$. Since $K_{\infty}(\phi[a]) \subseteq K_{\infty}\left(\Lambda_{\phi, \infty}\right)$, we obtain:

Theorem 5.2. Let $\phi$ be a Drinfeld $A$-module of rank 2 over $K$ and $\mathfrak{D}(K(\phi[a]) / K)$ be the different divisor of $K(\phi[a]) / K$. Then

$$
\begin{aligned}
\operatorname{deg}_{K} \mathfrak{D}(K(\phi[a]) / K) \leq & 2 \operatorname{deg}_{K} a+\operatorname{deg}_{K} \eta+\frac{2}{q-1} \operatorname{deg}_{K} \delta \\
& +v_{\infty}\left(\mathcal{D}\left(K_{\infty}\left(\Lambda_{\phi, \infty}\right) / K_{\infty}\right)\right),
\end{aligned}
$$

where $\delta$ is the (monic) denominator of $j(\phi)$ as represented by a fraction in reduced form, and $\eta$ is the product of finite primes $\mathfrak{p}$ such that $\phi$ has bad reduction over $K_{\mathfrak{p}}$.

This combined with Theorem4.1 gives an explicit bound on $\operatorname{deg}_{K} \mathfrak{D}(K(\phi[a]) / K)$ in terms of $j(\phi)$, the primes of bad reduction of $\phi$, and $a$.

\section{ACKNOWLEDGEMENTS}

We would like to express our appreciation to the referees of this paper for their valuable suggestions. Their careful reading of our paper corrected errors, made significant contributions to its content, and resulted in the current approach taken. In particular, we are indebted to the referees who explained the relevance of Gekeler's work and brought to our attention Rosen's paper [15].

\section{REFERENCES}

[1] I. Chen and Y. Lee, Coefficients of exponential functions attached to Drinfeld modules of rank 2, to appear, Manuscripta Math., DOI:10.1007/s00229-011-0505-2, 2012.

[2] C. David, Frobenius distributions of Drinfeld modules of any rank, J. Number Theory, 90, no. 2 (2001), 329-340. MR1858082 (2002k:11084)

[3] F. Gardeyn, Openness of the Galois image for $\tau$-modules of dimension 1, J. Number Theory, 102, no. 2 (2003), 306-338. MR1997794 (2005a:11083)

[4] F. Gardeyn, Une borne pour l'action de l'inertie sauvage sur la torsion d'un module de Drinfeld, Arch. Math., 79 (2002), 241-251. MR.1944948(2003i:11073)

[5] F. Gardeyn, t-motives and Galois representations, PhD thesis, ETH Zurich, 2001. $\operatorname{MR} 2715545$ 
[6] E. Gekeler, A survey on Drinfeld modular forms, Turkish J. Mathematics, 23, no. 4 (1999), 485-518. MR:1780937 (2001f:11071)

[7] E. Gekeler, Para-Eisenstein series for the modular group GL $\left(2, \mathbb{F}_{q}[T]\right)$, Taiwanese J. Math., 15, no. 4 (2011), 1463-1475. MR2848968

[8] E. Gekeler, On the Drinfeld discriminant function, Compositio Mathematica, 106 (1997), 181-202. MR1457338 (98e:11071)

[9] E. Gekeler, Zero distribution and decay at infinity of Drinfeld modular coefficient forms, Int. J. Number Theory, 7 (2011), 671-693. MR2805575

[10] D. Goss, Basic structures of function field arithmetic, Springer-Verlag, Berlin, 1996. MR.1423131 (97i:11062)

[11] R. Pink and M. Traulsen, The isogeny conjecture for $t$-motives associated to direct sums of Drinfeld modules, J. Number Theory, 117, no. 2 (2006), 355-375. MR2213770 (2007b:11087)

[12] R. Pink and M. Traulsen, The Galois representations associated to a Drinfeld module in special characteristic. III. Image of the group ring, J. Number Theory, 116, no. 2 (2006), 373-395. MR2195931 (2006k:11109)

[13] R. Pink, The Galois representations associated to a Drinfeld module in special characteristic. II. Openness, J. Number Theory, 116, no. 2 (2006), 348-372. MR2195930 (2006k:11108)

[14] R. Pink, The Galois representations associated to a Drinfeld module in special characteristic. I. Zariski density, J. Number Theory, 116, no. 2 (2006), 324-347. MR2195929 (2006k:11107)

[15] M. Rosen, Formal Drinfeld modules, J. Number Theory, 103, no. 2 (2003), 234-256. MR2020270 (2004j:11056)

[16] J.-P. Serre, Corps locaux, Paris, 1968. MR0354618 (50:7096)

[17] Y. Taguchi, Ramifications arising from Drinfeld modules, The arithmetic of function fields (Columbus, Ohio, 1991), 171-187, Ohio State Univ. Math. Res. Inst. Publ., 2, de Gruyter, Berlin, 1992. MR1196519 (94b:11049)

[18] Y. Taguchi, Semi-simplicity of the Galois representations attached to Drinfeld modules over fields of "infinite characteristics", J. Number Theory, 44, no. 3 (1993), 292-314. MR.1233291 (94k:11064)

Department of Mathematics, Simon Fraser University, Burnaby, British Columbia, CANADA V5A 1S6

E-mail address: ichen@math.sfu.ca

Department of Mathematics, Ewha Womans University, Seoul, 120-750, Republic of Korea

E-mail address: yoonjinl@ewha.ac.kr 